\title{
Dual-band binary metamaterial absorber based on low-permittivity all-dielectric resonance surface
}

Qiang Wang ${ }^{1, *}$, Fen Zhang ${ }^{2, *}$, Yijun Xiong ${ }^{1}$, Yan Wang ${ }^{1}$, Xiu-Zhi Tang ${ }^{1}$, Chao Jiang ${ }^{1, b)}$, Isaac Abrahams ${ }^{3}$, Xiaozhong Huang ${ }^{1, a)}$

${ }^{13}$ Hunan Key Laboratory of Advanced Fibers and Composites, School of Aeronautics and Astronautics, Central South University, Changsha, 410083, China

${ }^{2}$ School of Physics and Electronics, Central South University, Changsha, 410083

${ }^{3}$ School of Biological and Chemical Sciences, Queen Mary University of London, London, E1 4NS, UK

*These authors contributed equally to this work.

${ }^{a)}$ E-mail: huangxzh@csu.edu.cn (X.Huang)

${ }^{b)}$ E-mail: jiangchao@csu.edu.cn (C.Jiang)

A binary-structured metamaterial absorber (BMA) consisting of a low-permittivity dual-layer all-dielectric resonance surface (ADRS) and reflector was simulated and experimentally validated. Analyses of relative impedance, electric/magnetic field and power loss density indicated that the proposed BMA exhibits two absorption peaks at $14.65 \mathrm{GHz}$ and $16.61 \mathrm{GHz}$, resulting from the magnetic and electrical responses of ADRS, respectively. The dependences of absorption properties on the dimensions of the ADRS and material parameters of the ADRS are discussed. It is concluded that the upper layer of the ADRS acts as an impedance-matching layer directly influencing the absorption intensity, while bottom layer offers frequency selectivity in the $13-15 \mathrm{GHz}$ range. The current design uses a low-permittivity ADRS, with simplified design and easy preparation and is notably different from conventional ternary-structured metamaterial absorbers based on a metallic resonance surface. The simplicity of the proposed BMA makes it a promising low-cost ambient 
temperature alternative to conventional metamaterial absorbers and could open up practical applications.

Keywords: Binary-structured metamaterial absorber; Low-permittivity all-dielectric resonance surface; Frequency selectivity; Simplified design and easy preparation 


\section{Introduction}

Metamaterial absorbers (MAs) have been widely studied because of their advantages of thinness, lightness, and adjustable absorbtion frequency compared to conventional absorbers. In particular, multi-band MAs are of interest for application in the fields of perfect lenses [1, 2], filters [3], resonators [4], antennae [5] and radio frequency absorbers [6].

Ternary-structured MAs comprising of a metallic resonance surface (MRS), dielectric and reflector have been widely reported [7-12]. In order to broaden the absorption bands, the MRS, an essential component to trigger the electric or magnetic resonance, usually has as a complicated multi-resonant structure such as multi-slit [11], checkerboard array [12], fractal [13], or concentric nested [14] architectures. However, the complexity of designs and difficulty of fabrication of metallic resonance surfaces have impeded the practical applications of MAs.

Recently, an all-dielectric resonance surface (ADRS) has been developed, suggesting that through special structural designs, high-permittivity all-dielectric resonators can exhibit negative electromagnetic (EM) permittivity and can trigger an electrical or magnetic response [15, 16]. Zhao et al. obtained isotropic negative permeability from a three-dimensional (3D) all-dielectric composite by arraying $\mathrm{BaSrTiO}_{3}$ (BST) cubes in a 3D Teflon substrate [16]. Apart from being employed in resonators, ADRS have also been used to construct a binary-structured metamaterial absorber (BMA), which was composed only of an ADRS and a reflector [17]. Liu et al. fabricated BMAs using a reflector and periodic ADRSs made of $\mathrm{SrTiO}_{3}$ [18] or BST based compositons [19]. In addition, some composite ADRSs composed of high-permittivity dielectrics inserted into an acrylonitrile butadiene styrene matrix substrate have been demonstrated in the fabrication of multiband BMAs [20]. Previous BMAs were based on high-permittivity ceramic ADRSs, with 
simplified designs and better high-temperature resistance, being suitable for high-temperature absorption applications. For lower temperature applications, simplified low-cost MAs could be used, but these have been rarely reported.

Through careful structural design, dielectric-air interfaces can be formed at the low-permittivity dielectric plate, resulting in an ADRS [21]. According to interference theory [22, 23], multiple reflection waves with different phase will be triggered when electromagnetic waves are incident on an ADRS, thereby resulting in multiple interferences and exciting electric or magnetic resonances [24]. Interestingly, the proposed low-permittivity ADRSs achieve dual-band and adjustable absorption by virtue of the structural design of the resonance surfaces in a similar way to conventional metallic resonance surfaces [7-12]. Additionally, the low-permittivity ADRSs are usually made from resins, which can be fabricated rapidly, assisted by mechanical engraving or 3D print technology $[24,25]$. This represents a potential method for fabrication of simplified, low-cost MAs for lower temperature applications.

We have previously demonstrated a BMA based on a low-permittivity ADRS and have explained how absorption peaks arise only by virtue of the structural design of the ADRS [26]. To further verify the feasibility of BMA, in the present work we adopt the general and simplified rectangular-hole design instead of complex multilateral-hole design [26]. In addtion, we extend these studies to focus on the frequency selection mechanism and the effects of material properties on the frequency selection characteristics, which are important considerations for MAs. Here we report on the design, simulation and fabrication of a BMA based on a dual-layer low-permittivity ADRS, which achieved two strong absorption peaks at 14.65 and $16.61 \mathrm{GHz}$, and good frequency selection characteristic. The wave-absorbtion mechanism is investigated through the relative impedance and 
analyzing power loss density (PLD) distributions and electric and magnetic-field distributions. The dependences of absorption properties on the dimensions of the ADRS and the material parameters are explained in detail.

\section{Design and simulation}

A schematic diagram showing a periodic unit of the optimized BMA model is given in Figure 1. The ADRS is composed of two dielectric layers, an upper layer (layer 1), with a rectangualar hole of dimensions $15 \mathrm{~mm} \times 7 \mathrm{~mm}$ and a bottom layer (layer 2) with a square-hole of dimensions $15 \mathrm{~mm} \times$ $15 \mathrm{~mm}$. Both layers have the same outer dimensions of $18 \mathrm{~mm} \times 18 \mathrm{~mm}$ and $1.5 \mathrm{~mm}$ thickness. Both are composed of a uniform epoxy resin with a permittivity $\varepsilon_{r}=4.3$, and a dielectric loss tangent $\tan \delta$ $=0.025$.

The CST Microwave Studio (CST MWS) software [27] was used for model building and the finite-difference time-domain method used in simulation. In the simulation, a periodic unit of the BMA was built in the CST MWS workspace. Both $\mathrm{x}$ and $\mathrm{y}$-directions were arranged with periodic boundary conditions, with an open boundary condition in z-direction. A transverse-electric (TE) -mode polarized plane electromagnetic wave was assumed in the simulation and propagated perpendicularly from the $\mathrm{z}+$ direction to the $\mathrm{x}-\mathrm{y}$ plane. Through the simulations the reflection coefficient $\left(\mathrm{S}_{11}\right)$, transmission coefficient $\left(\mathrm{S}_{21}\right)$ and some important representations, including PLD distributions and electric/magnetic-field distributions were obtained. The absorptivity as a function of frequency, $A(\omega)$, can be calculated using:

$$
A(\omega)=1-R(\omega)-T(\omega)
$$


where, $R(\omega)$ is the reflectivity and is equal to $\left|S_{11}\right|^{2}$, and $T(\omega)$ is transmittivity and is equal to $\left|S_{21}\right|^{2}$. A perfect electric conductor (PEC) was assumed in the simulations and thus $S_{21}=0$.

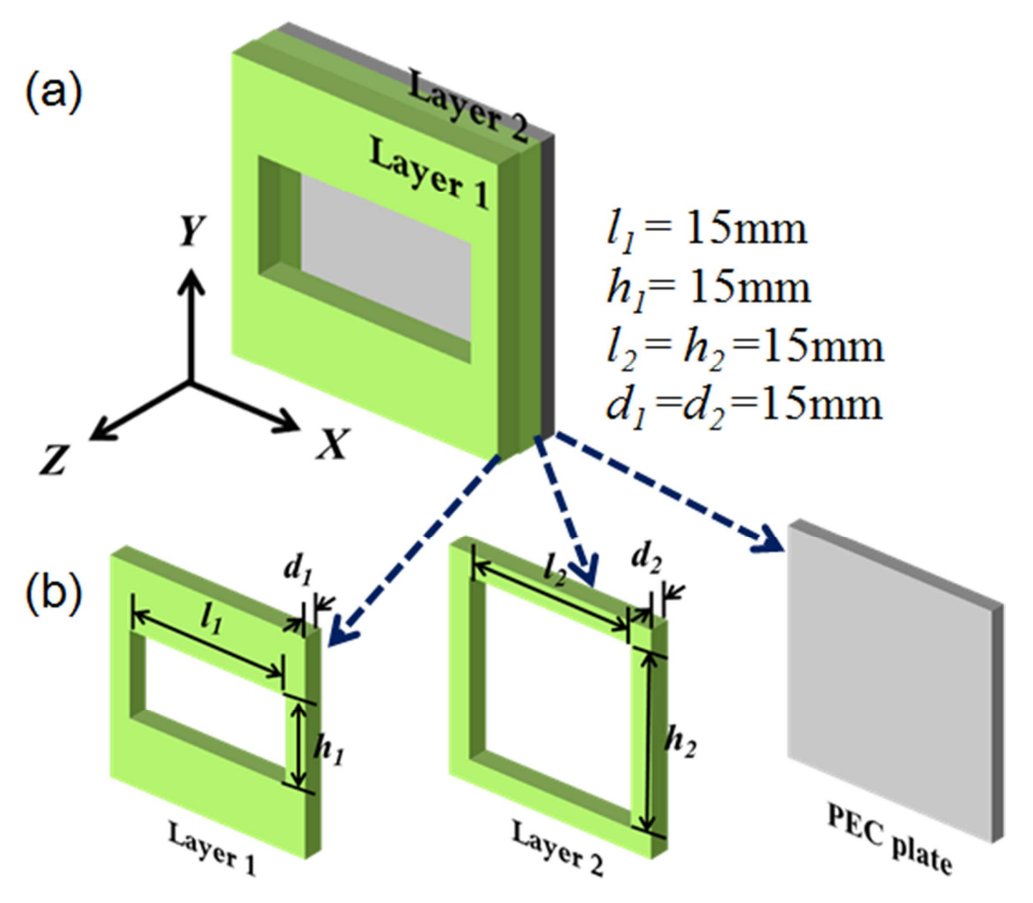

Fig. 1. Schematic showing the studied BMA construction: overall schematic (a) and (b) separate schematics.

The reflection/absorption results of the BMA in the frequency range 12-18 GHz are presented in Figure $2 \mathrm{a}$ and indicate that the BMA exhibits two absorption peaks at 14.65 and $16.61 \mathrm{GHz}$, with high absorbtivities of $99.78 \%$ and $99.95 \%$, respectively. The relative impedance of the BMA calculated using formula (2) [28] is shown in Figure 2b. It can be found that around $14.65 \mathrm{GHz}$ and $16.61 \mathrm{GHz}$, the real part of $\mathrm{Z}(\omega)$ approaches 1 and the imaginary part is approximately 0 . Thus, the BMA achieves perfect impedance matching with free space at two resonance frequencies.

$$
\mathrm{z}(\omega)=\sqrt{\frac{\left(1+S_{11}\right)^{2}-S_{21}{ }^{2}}{\left(1-S_{11}\right)^{2}-S_{21}{ }^{2}}}
$$



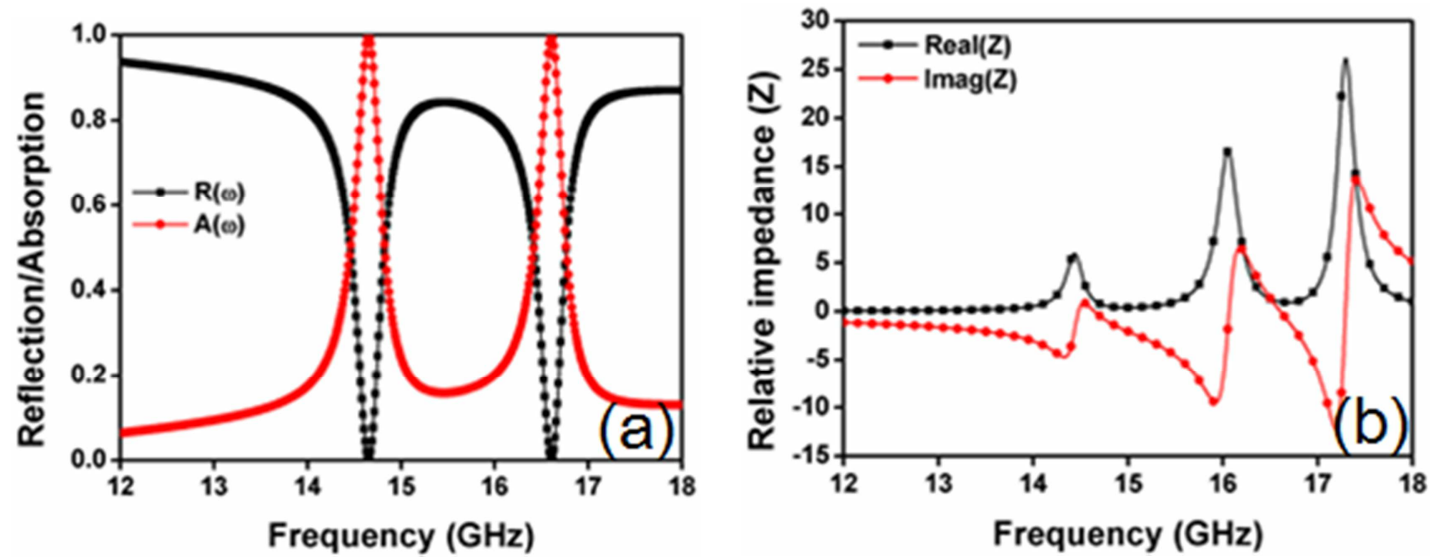

Fig. 2. Simulated results of (a) reflection $(R(\omega))$ and absorption $(A(\omega))$, and (b) real part (Real (Z)) and imaginary part (Imag $(Z))$ of relative impedance of the studied BMA.

\section{Analysis and discussion}

\subsection{Studies on the resonance mechanism of the studied BMA}

In order to investigate microwave loss mechanisms of the BMA at two resonance frequencies, the dynamic PLD distributions were simulated and are presented in Figure 3. The PLD distributions on the E-o-H and E-o-K planes (where $\mathrm{E}$ is the electric field direction, $\mathrm{H}$ is the magnetic field direction and $\mathrm{K}$ is the microwave vector), as shown in Figures $3 \mathrm{a}$ and $3 \mathrm{~b}$, respectively, indicate that the strong power loss at $14.65 \mathrm{GHz}$ is primarily associated with the areas in layer 2 that lie under the rectangular holes in layer 1. Figures $3 \mathrm{c}$ and $3 \mathrm{~d}$ show PLD results for the E-o-H and H-o-K planes at 16.61 GHz. Here the power loss is concentrated on the corners and the middle of layer1. 
(a)

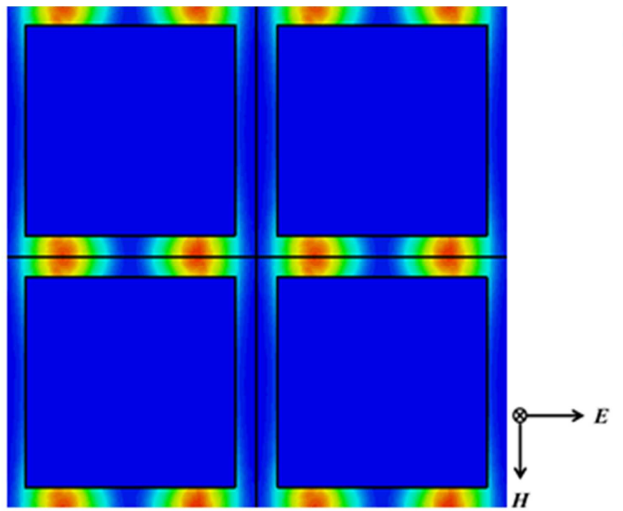

(b)

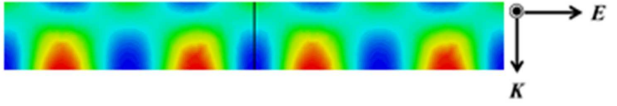

(c)

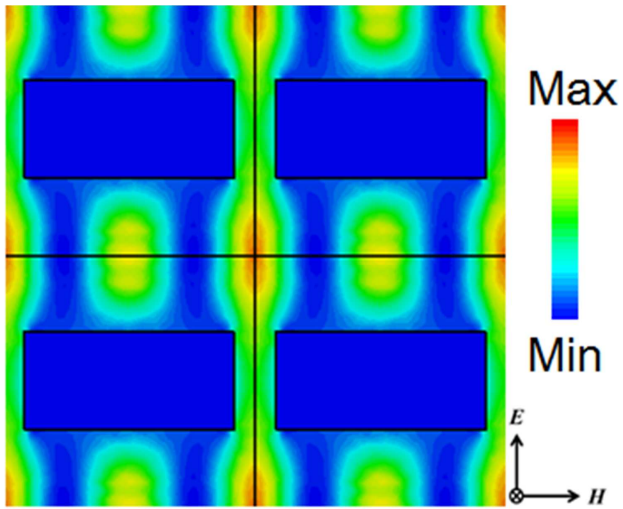

(d)

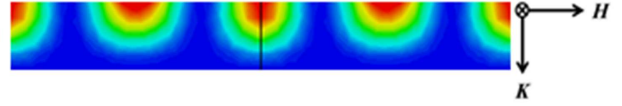

Fig. 3. PLD distributions for the studied BMA: distributions at $14.65 \mathrm{GHz}$ on (a) E-o-H plane and (b) E-o-K plane, with a maximum PLD of $6.02 \times 10^{6} \mathrm{~W} / \mathrm{m}^{3}$; and at $16.61 \mathrm{GHz}$ on (c) E-o-H plane and (d) H-o-K plane, with a maximum PLD of $3.74 \times 10^{6} \mathrm{~W} / \mathrm{m}^{3}$.

(a)

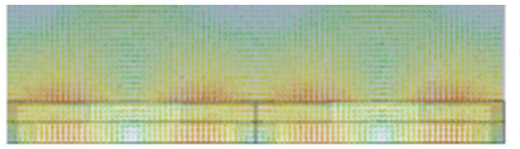

(b)

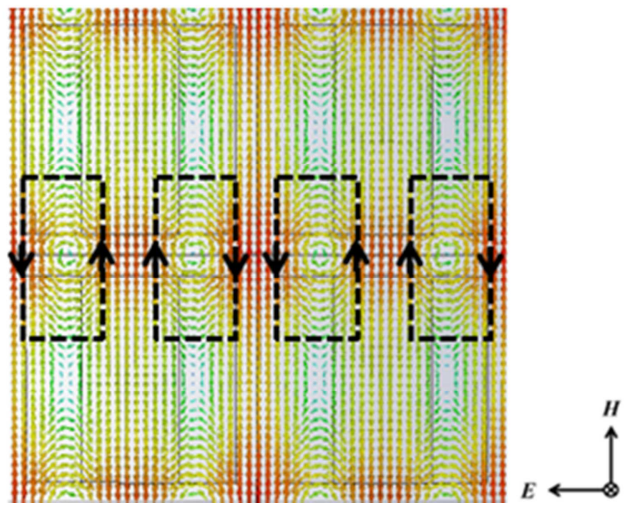

(c)

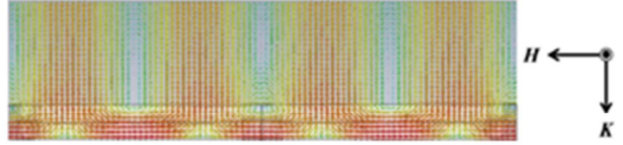

(d)

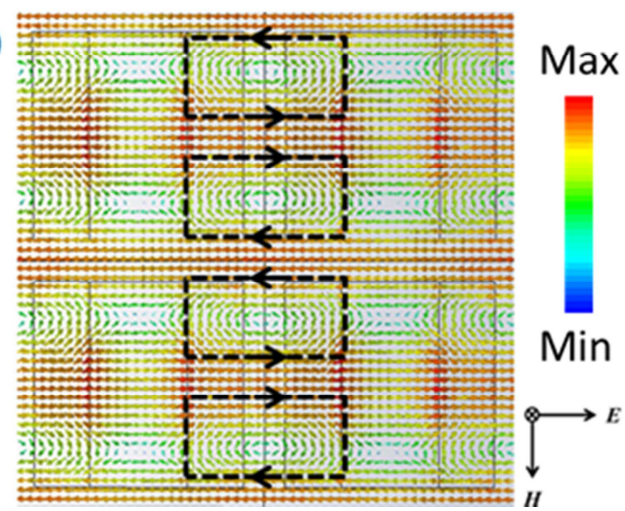

Fig. 4. Field distributions of the studied BMA on the surface of layer 2 at $14.65 \mathrm{GHz}$, showing (a) the electric-field distribution on the E-o-K plane and (b) the magnetic-field distribution on the E-o-H plane, with a maximum electric intensity of $1.35 \times 10^{4} \mathrm{~V} / \mathrm{m}$ and a maximum magnetic intensity of 67.47 A/m; and at 16.61 GHz, showing (c) the magnetic-field distribution on the H-o-K plane and (d) the electric-field distribution on the E-o-H plane, with a maximum electric intensity of $1.111 \times 10^{4}$ $\mathrm{V} / \mathrm{m}$ and a maximum magnetic intensity of $44.37 \mathrm{~A} / \mathrm{m}$. The dashed boxes represent inverted electric- or magnetic-field rings.

To further understand the resonance mechanisms of the two absorption peaks, the dynamic distributions of electric and magnetic-fields were monitored on the surface of layer 2 and are presented in Figure 4. At $14.65 \mathrm{GHz}$, the configuration of magnetic-field (Figure 4b) is accompanied 
by some inverted magnetic-field rings (dashed box) indicative of electric dipoles. Electric dipole oscillations result in a strong electric response of the BMA to incident electric field (Figure 4a). Figures $4 \mathrm{c}$ and $4 \mathrm{~d}$ show the magnetic-field distribution on the $\mathrm{H}-\mathrm{O}-\mathrm{K}$ plane and electric-field distribution on E-o-H plane, respectively, at $16.61 \mathrm{GHz}$. It can be discerned that the enhanced reversed electric-field lines, which are parallel to the E vector appear in the middle and boundaries of the unit (Figure 4d), resulting in the generation of inverted electric-field rings (dashed box). Due to the displacement current between these lines, the electric-field configuration can be viewed as arising from magnetic dipoles, causing an extensive magnetic response of the BMA to the incident magnetic-field (Figure 4c). It can be viewed as a breakthrough which is differ from the conclusion in [26] that all absoprtion peaks derive from electric resonances, which means that like traditional MRS, the ADRS can excite both electric and magnetic resonances, even if they exist simultaneously.

\subsection{Studies on frequency selective characteristics}

The dependence of absorption properties on the dimensions of the holes arrays $\left(l_{1}, h_{1}, l_{2}, h_{2}\right)$ was studied. As shown in Figures $5 \mathrm{a}$ and $5 \mathrm{~b}$, on decreasing $l_{l}$ (from 15 to $7 \mathrm{~mm}$ ) or increasing $h_{1}$ (from 7 to $15 \mathrm{~mm}$ ), the two resonance peaks shift slightly accompanied by a significant decrease in reflection loss and demonstrates that the dimensions $\left(l_{1}, h_{1}\right)$ of the rectangular-hole array in layer1 strongly influence the attenuation of the incident EM wave. Layer1 as the upper unit can be viewed as an impedance-matching layer, whose dimensions and permittivitty dictate the degree of impedance-matching between the BMA and free space [29]. In the case of determining the material, unit sizes, and the thickness of ADRSs, scaling directly affects absorption properties. Structural alteration of layer1 can reduce the quality of impedance-matching, resulting in lower absorption. 

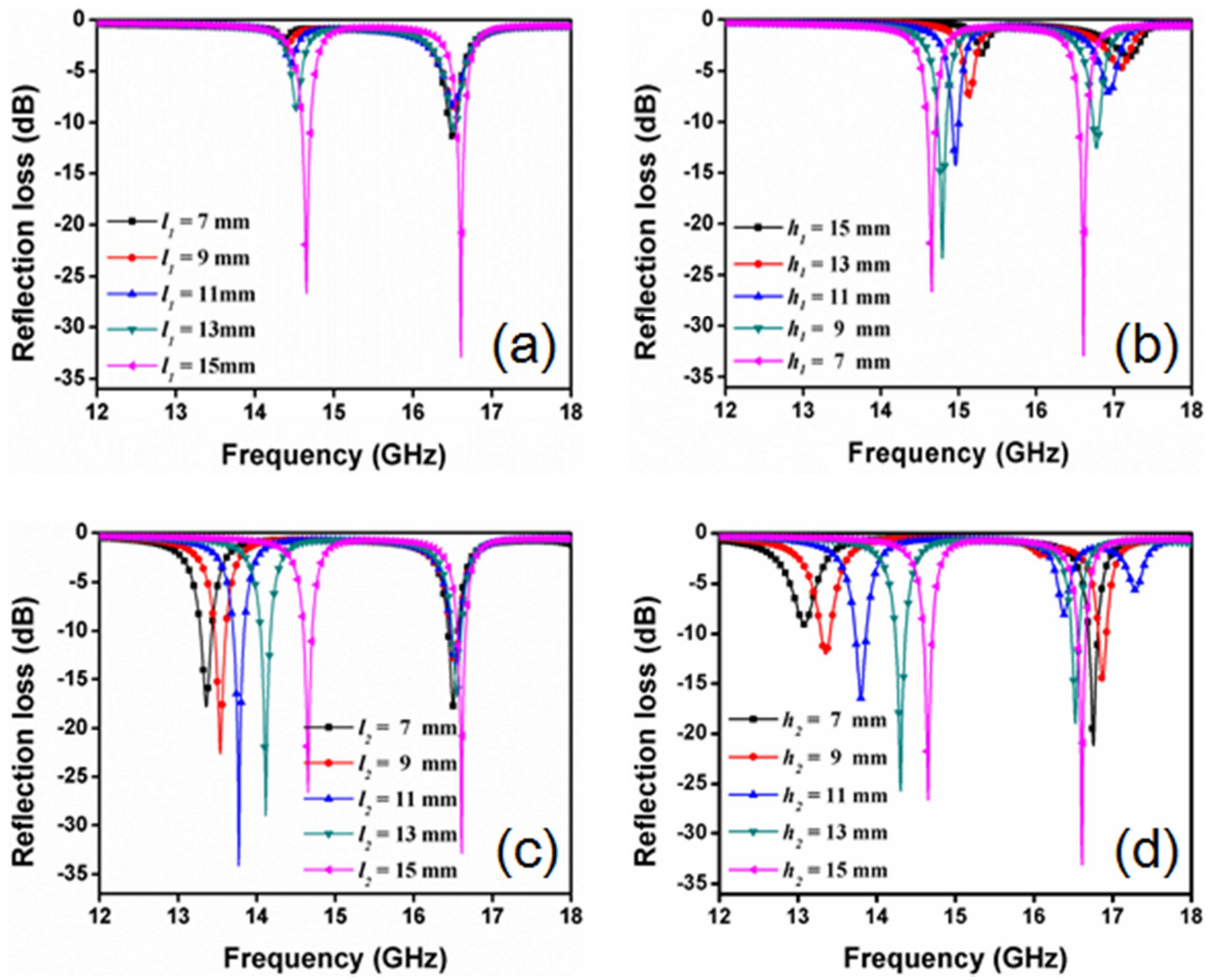

Fig. 5. Reflection loss curves of studied BMA with different perforation dimensions showing dependence on: (a) length $l_{1}$ and (b) height $h_{1}$ of the hole in layer1; and (c) length $l_{2}$ and (d) $h_{2}$ of the hole in layer 2 .

For layer 2 (Figures $5 \mathrm{c}$ and 5d), as the values of $l_{2}$ or $h_{2}$ increase from $7 \mathrm{~mm}$ to $15 \mathrm{~mm}$, the first resonance peak shifts towards higher frequency, suggesting that the dimensions $\left(l_{2}, h_{2}\right)$ of the rectangular-hole array in layer 2 determine the first resonance frequency. As discussed above (section 3.1) the low frequency resonance peak is associated with electric dipole oscillation. Based on electric dipole resonance theory $[30,31]$, the electric dipole resonance frequency is inversely proportional to the effective permittivity when the structure dimensions are fixed. The effective permittivity of layer 2 decreases as $l_{2}$ or $h_{2}$ increase from $7 \mathrm{~mm}$ to $15 \mathrm{~mm}$, leading to the absorption peak shifting towards higher frequency.

Figures $5 \mathrm{~b}$ and $5 \mathrm{~d}$, show a very small frequency-shift around $16.61 \mathrm{GHz}$ when the values of $h_{1}$ 
or $h_{2}$ are changed. In the contrast, no shift for this absorption peak is observed with variation of $l_{l}$ or $l_{2}$ (Figures 5a and 5c). The reversed electric-field lines as presented in Figure 4c form oscillating magnetic dipoles triggering the magnetic resonance. For the current BMA, the resonance surface consists of an all-dielectric material. Therefore, dimensional changes along the E vector $\left(h_{1}\right.$ or $\left.h_{2}\right)$ have a little influence on interference with the electric-field, resulting in a small frequency shift at the second resonance frequency, while variations along the $\mathrm{H}$ vector $\left(l_{1}\right.$ or $\left.l_{2}\right)$ have little effect on the electric-field behavior.

\subsection{Dependence of absorbing properties on material parameters}

The material parameters of the ADRS also need to be considered as they affect the reflection loss of the incident EM wave. Using the optimized parameters for the BMA shown in Figure 1, the dependences of reflection loss on permittivity $\varepsilon_{r}$ and dielectric loss ( $\tan \delta$ ) of the ADRS were calculated (Figure 6). As shown in Figure 6a, the double absorption peaks move to lower frequency when the permittivity $\varepsilon_{r}$ varies from 3.8 to 5.8 ; $\tan \delta$ remains unchanged. This can be explained by equation (3) based on the destructive interference theory [22]:

$$
n \cdot d=\frac{m \cdot c}{4 f_{0}}
$$

where $n$ and $c$ represent the refractive index and the light speed; $m$ is any positive odd integer (implying periodic anti-reflection); $d$ and $f_{0}$ are the thickness of dielectric and resonance frequency, respectively. For the current design, $m, c, d$ are all constants, hence, $f_{0}$ and $n$ are inversely proportional. Since the refractive index, $n$, for a dielectric material is directly proportional to the permittivity $\varepsilon_{r}$, according to Maxwell's electromagnetic theory, $\varepsilon_{r}$ and $f_{0}$ are also inversely proportional. As seen in Figure 6b, there is no frequency shift in the absorption peaks when $\tan \delta$ 
varies from 0.025 to 0.005 with $\varepsilon_{r}=4.3$, but there is a dramatic decrease in reflection loss. This is because the ADRS, as the exclusive resonant component, is an all-dielectric, and the EM wave attenuation is primarily due to the dielectric loss of material.
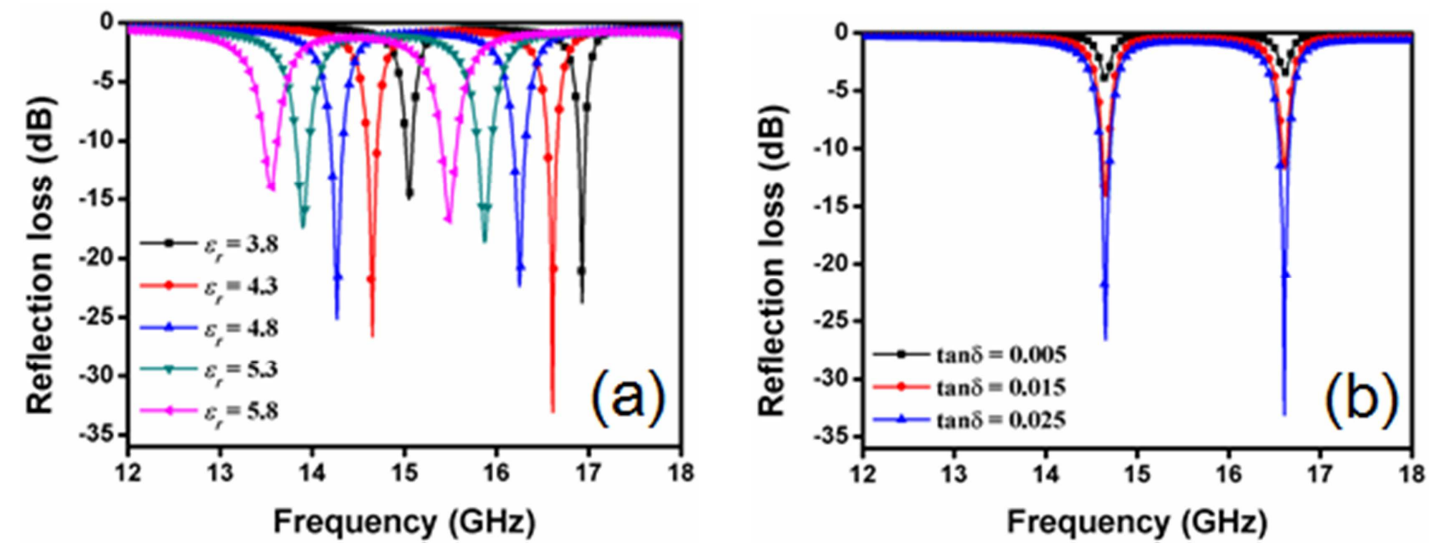

Fig. 6. Reflection loss curves of the studied BMA with different material parameters: (a) dependence on $\varepsilon_{r}$ of dielectric with $\tan \delta=0.025$ and (b) dependence on $\tan \delta$ of dielectric with $\varepsilon_{r}=4.3$.

\section{Experimental verification}

To validate the design presented in the current work, a specimen BMA of size of $180 \mathrm{~mm} \times 180$ mm was fabricated using a computer numerical controlled (CNC) engraving machine (which was produced by Jinan Sanqiang CNC Equipment Co. China). The preparation process diagram for the specimen is presented in Figure 7. In accordance with the tracks marked as dashed lines in Figure 7, an epoxy resin (FR4) plate with a thickness of $3 \mathrm{~mm}$ was carved twice, each with a carving depth of $1.5 \mathrm{~mm}$ to form the ADRS. The front and rear pictures of prepared ADRS are presented in Figure 8a. The obtained ADRS with an aluminum back plate were illuminated with a TE-mode polarized EM wave over the frequency range 12-18 GHz. The measurement was carried out using an NRL-arch reflectivity test system with a vector network analyzer (AV3629) in an EM anechoic chamber (The whole testing was provided by Hunan Boxiang new materials co. LTD, China). The measured and simulated reflection loss results are shown in Figure $8 \mathrm{~b}$ indicating that measured profile includes two 
absorption peaks around 14.6 and $16.4 \mathrm{GHz}$, entirely consistent with the simulation.

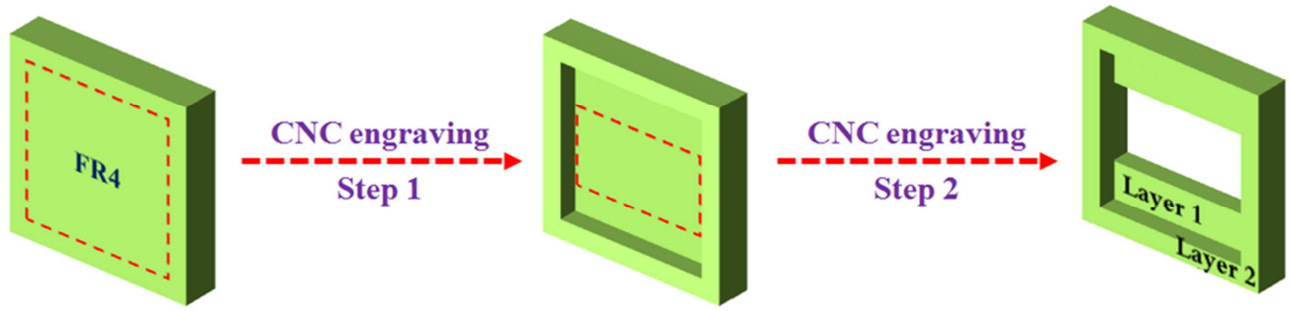

Fig. 7. Preparation process diagram of the ADRS specimen. The dashed lines indicate the engraving tracks during the preparation process.
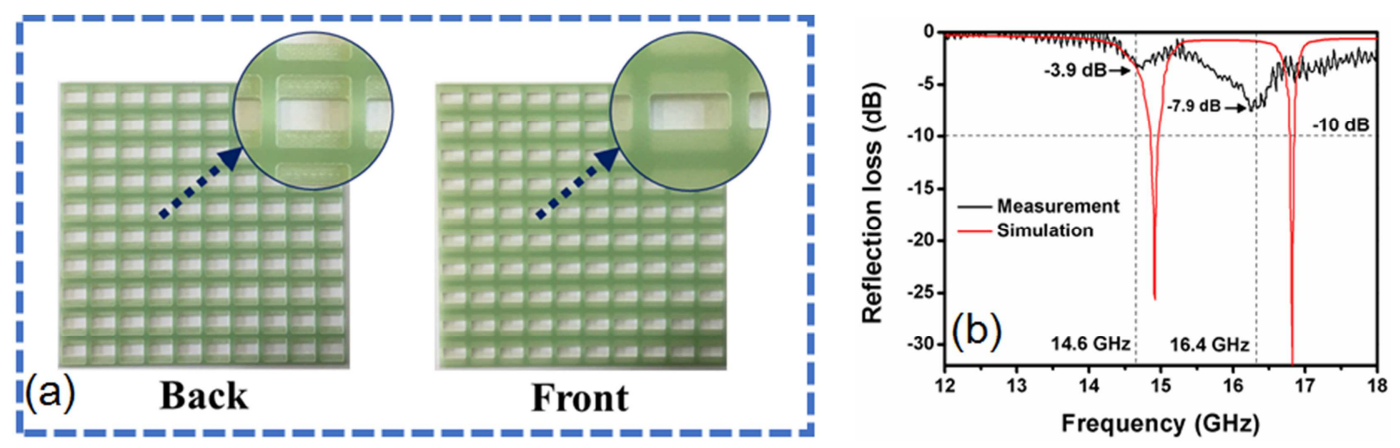

Fig. 8. (a) Front and back views of the prepared ADRS and (b) measured and simulated reflection loss curve for the prepared BMA over the frequency range $12-18 \mathrm{GHz}$.

The measured reflection loss at $14.6 \mathrm{GHz}$ is $-3.9 \mathrm{~dB}$, while that at $16.4 \mathrm{GHz}$ is $-7.3 \mathrm{~dB}$, which are smaller than seen in the simulations. It should be remembered here that the simulated refection loss was based on an infinite periodic structure, while only a limited specimen size could be fabricated and tested experimentally due to the practical limitations. In addition, the slight difference in the frequency of the second resonance between the experiment and simulation is likely due to the experimental uncertainties in fabrication and measurement, such as machining fillet errors, test errors, as well as data fitting errors. Although like this, we believe the current measured result that maximum value approaches to $-10 \mathrm{~dB}$ is a big plus and enough to exhibit the novelty and practical feasibility of proposed BMA, which uses low-permittivity all-dielectric resonance surface with low cost and easy preparation. 


\section{Conclusions}

In summary, a BMA based on a low-permittivity dual-layer ADRS has been designed, simulated and fabricated. The device exhibited two absorption peaks at $14.65 \mathrm{GHz}$ and $16.61 \mathrm{GHz}$ caused by electric resonance and magnetic resonance, respectively. The upper layer of the ADRS causes the higher frequency resonance at $16.61 \mathrm{GHz}$ and acts as an impedance matching layer, thereby greatly affecting the reflection loss of the BMA. The bottom layer of the ADRS induces a resonance at 14.65 $\mathrm{GHz}$ and allows the BMA to achieve frequency selectivity in the range 13-15 GHz. In contrast to other BMAs based on conventional metallic resonance surfaces, we have successfully used a low-permittivity ADRS to achieve dual-band absorption and frequency selectivity. Notably, the proposed BMA is easy to prepare and low-cost, which are significant factors in considering these devices for practical applications.

\section{Acknowledgements}

The work was supported by the National Defense Science and Technology Innovation Project Grant No. 1716313ZT01002601 and Grant No. 1716313ZT009052001; and the Science and Technology Plan Project of Hunan Province Grant No. 2015TP1007; Initial Research Funding for Special Associate Professor by Central South University Grant No. 502045002.

\section{References}

[1] J. B. Pendry, Phys. Rev. Lett. 85, 3966 (2000).

[2] N. Fang, H. Lee, C. Sun, and X. Zhang, Science 308, 534 (2005).

[3] J. Garcia-Garcia, J. Bonache, I. Gil, F. Martin, M. C. Velazquez-Ahumada, and J. Martel, IEEE Trans. Microw. Theory Tech. 54, 2628 (2006).

[4] M. L. Si, and X. Lv, Prog. Electromagn. Res. 83, 133 (2008).

[5] A. Turkmen, E. Ekmekci, and G. Turhan-Sayan, IET Microw. Antennas Propag. 6, 1102 (2012).

[6] F. Costa, S. Genovesi, and A. Monorchio, IEEE Trans. Microw. Theory Tech. 61, 146 (2013).

[7] N. L.Landy, S. Sajuyigbe, J. J. Mock, D. R. Smith, and W. J. Padilla, Phys. Rev. Lett. 100, 207402 (2008).

[8] H. Xiong, M. C. Tang, and J. S. Hong, J. Appl. Phys. 117, 207402 (2015). 
[9] X. J. Huang, H. L.Yang, S. Q. Yu, J. X. Wang, M. H. Li, and Q. W. Ye, J. Appl. Phys. 113, 213516 (2013).

[10] S. Bhattacharyya, and K. V. Srivastava, J. Appl. Phys. 115, 064508 (2014).

[11]Q. W. Ye, Y. Liu, H. Lin, M. H. Li, and H. L. Yang, Appl. Phys. A 107, 155 (2012).

[12] J. W. Park, P. V. Tuong, J. Y. Rhee, K. W. Kim, W. H. Jang, E. H. Choi, L. Y. Chen, and Y. P. Lee, Opt. Express 21, 9691 (2013).

[13]H. X. Xu, G. M. Wang, M. Q. Qi, J. G. Liang, J. Q. Gong, and Z. M. Xu, Phys. Rev. B 86, 3368 (2012).

[14]L.Huang, and H. Chen, Prog. Electromagn. Res. 113, 103 (2011).

[15]L. Peng, L. X. Ran, H. S. Chen, H. F. Zhang, J. A. Kong, and T. M. Grzegorczyk, Phys. Rev. Lett. 98, 57403 (2007).

[16]Q. Zhao, L. Kang, B. Du, H. Zhao, Q. Xie, X. Huang, B. Li, J. Zhou, and L. Li, Phys. Rev. Lett. 101, 027402 (2008).

[17] N. V. Dung, B. S. Tung, B. X. Khuyen, Y. J. Yoo, and Y. P. Lee, J. Korean Phys. Soc. 68, 1008 (2016).

[18]X. M. Liu, Q. Zhao, C. W. Lan, and J. Zhou, Appl. Phys. lett. 103, 031910 (2013 ).

[19]X. Liu, K. Bi, B. Li, Q. Zhao, and J. Zhou, Opt. Express 24, 20454 (2016).

[20]X. M. Liu, C. W. Lan, K. Bi, B. Li, Q. Zhao, and J. Zhou, Appl. Phys. lett. 109, 062902 (2016).

[21]F. Yu, J. Wang, J. F. Wang, H. Ma, H. L. Du, Z. Xu, and S. B. Qu, Appl. Phys. Lett. 107, 211906 (2015).

[22]J. B. Sun, L. Y. Liu, G. Y. Dong, and J. Zhou, Opt. Express 19, 21155 (2011).

[23]T. L. Wanghuang, W. J. Chen, Y. J. Huang, and G. J. Wen, AIP Adv. 3, 102118 (2013).

[24]D. V. Isakov, Q. Lei, F. Castles, C. J. Stevens, C. R. M. Grovenor, and P. S. Grant, Mater. Design 93, 423 (2016).

[25]F. Castles, D. Isakov, A. Lui, Q. Lei, C. E. J. Dancer, Y. Wang, J. M. Janurudin, S. C. Speller, C. R. M. Grovenor, and P. S. Grant, Scientific Reports, 622714 (2016).

[26]Q. Wang, Y. Wang, X. Z. Tang,X. Z. Huang, Y. J. Xiong, and F. Zhang, J. Adv. Dielectrics 8, 1850021 (2018).

[27]R. E. Jones, F. Simonetti, and I. P. Bradley, J. Nondestruct. Eval. 31, 117 (2012).

[28]D. R. Smith, D. C. Vier, Th.Koschny, and C. M. Soukoulis, Phys. Rev. E 71, 036617 (2005).

[29]Z. Ma, C. T. Cao, Q. F. Liu, and J. B. Wang, Chinese Phys. Lett. 29, 38401 (2012).

[30]Q. Wang, X. Z. Tang, D. Zhou, Z. J. Du, and X. Z. Huang, IEEE Antenn. Wirel. Propag. Lett. 16, 3200 (2017).

[31]W. R. Holland, and D. G. Hall, Phys. Rev. Lett. 52, 1041 (1984). 